\title{
Primary Mixed Adenoneuroendocrine Carcinoma (MANEC) of Gallbladder, Report of Two Cases with Different Histologic and Immunohistochemical Features
}

\author{
Cyrus Parsa ${ }^{1^{*}}$, Robert Orlando ${ }^{2}$, Krishna Narayanan $^{3}$, David Duate ${ }^{4}$ and Shaun Webb ${ }^{5}$ \\ ${ }^{1}$ Department of Pathology, Western University of Health Sciences, Pomona, California and Director of Laboratories, Beverly Hospital, Montebello, California, US \\ ${ }^{2}$ Department of Pathology, Beverly Hospital, Montebello, California, US \\ ${ }^{3}$ Department of Surgery, Beverly Hospital, Montebello, California, US \\ ${ }^{4}$ Department of Surgery, Beverly Hospital, Montebello, California, US \\ ${ }^{5}$ Fourth Year Medical Student at Western University of Health Sciences, Pomona, California, US
}

*Corresponding author: Cyrus Parsa, Chair, Department of Pathology, Western University of Health Sciences, Pomona, California and Director of Laboratories, Beverly Hospital, Montebello, California, US, Tel: 323-889-2414; Fax: 323-889-2406; E-mail: cparsa@westernu.edu

Received Date: March 10, 2018; Accepted Date: May 22, 2018; Published Date: May 26, 2018

Copyright: (c) 2018 Parsa C, et al. This is an open-access article distributed under the terms of the Creative Commons Attribution License, which permits unrestricted use, distribution, and reproduction in any medium, provided the original author and source are credited.

\begin{abstract}
Since implementation of immunohistochemistry, the occurrence of neuroendocrine cells in adenocarcinomas of gastrointestinal tract has been well documented and is not uncommon. The mixture of neuroendocrine carcinoma and adenocarcinoma (MANEC), where each represents at least $30 \%$ of the neoplasm, is however very uncommon and occurs mostly in the stomach, appendix, and large intestine. Their occurrence in the gallbladder, as the primary site, is exceptionally rare. We present two elderly patients, one male and one female with clinical manifestations of cholecystitis. The cholecystectomy specimens from these patients revealed MANEC. In one patient, the epithelial component was conspicuously papillary and well-differentiated. In our second case, the epithelial component was variably poorly differentiated carcinoma with mixed neuroendocrine collision features. In this paper we describe the clinicopathologic features of these two cases with emphasis on differences in their gross, histologic, and immunohistochemical findings. These differences may be attributed to the proposed varied pathways for the origin of neuroendocrine cells in the gallbladder.
\end{abstract}

\section{Introduction}

Mixed adenoneuroendocrine carcinoma (MANEC), described and recognized by WHO in 2010, is a rare gastrointestinal neoplasm [1], characterized by the presence of at least $30 \%$ each of epithelial and neuroendocrine components [2]. The tumor may appear in various levels of the digestive tract including the oesophagus, stomach, colon and appendix, as well as other sites, such as the bladder. MANEC is an exceptionally rare neoplasm of gallbladder. The neoplasm occurs mostly in the middle-aged to elderly females with mean age of 64 years. Based on few case studies, suggested pathogenesis includes intestinal metaplasia-dysplasia-carcinoma sequence or a neoplastic stem cell with potential for transformation along several tissue cell lines. The prognosis is generally poor unless early cholecystectomy is performed and MANEC is an incidental finding.

\section{Case Reports}

\section{Case 1}

An obese 74-year-old female with history of hypertension and hypercholesterolemia presented with complaints of general malaise and anorexia of two weeks duration. She had a single brief episode of syncope while at a shopping mall a few days preceding her presentation. One other episode of syncope was reported 2 years prior, for which the patient was treated conservatively.
The patient also admitted to a history of peptic ulcer disease which was treated medically, and a history of vague abdominal pain. At the time of clinical presentation, the patient denied headache, nausea, abdominal pain, abdominal distention, vomiting, blurring of vision, changes in weight or in bowel habits. The patient denied history of cardiovascular disease or diabetes. She had a breast-cyst removed many years ago which was found to be benign. The patient's family history was unremarkable with no significant health concerns. The patient denied smoking or drinking alcohol.

Upon physical examination, the abdomen was negative for masses or tenderness on palpation with no rebound tenderness or guarding. A $\mathrm{CT}$ of the abdomen and pelvis was performed, which showed sludge in the gallbladder. Ultrasound revealed an indeterminate mass in the gallbladder, and a follow-up MRI showed a $2.7 \times 4.2 \times 1.7 \mathrm{~cm}$ enhancing mass extending from the floor of the gallbladder fundus. There was no evidence for common bile duct or intrahepatic duct dilatation. The patient underwent a laparoscopic cholecystectomy with a wedge resection of the liver.

The gallbladder was $8 \mathrm{~cm}$ long and up to $4 \mathrm{~cm}$ in circumference. The serosal surface was predominantly pink brown and smooth. The cut surface of the gallbladder revealed a polypoid mass in the fundic region, $2.0 \times 1.5 \mathrm{~cm}$ in greatest dimensions (Figure 1). 
Citation: Parsa C, Orlando R, Narayanan K, Duarte D, Webb S, (2018) Primary Mixed Adenoneuroendocrine Carcinoma (MANEC) of Gallbladder, Report of Two Cases with Different Histologic and Immunohistochemical Features. J Tumor Res \& Reports 3: 120.

Page 2 of 5

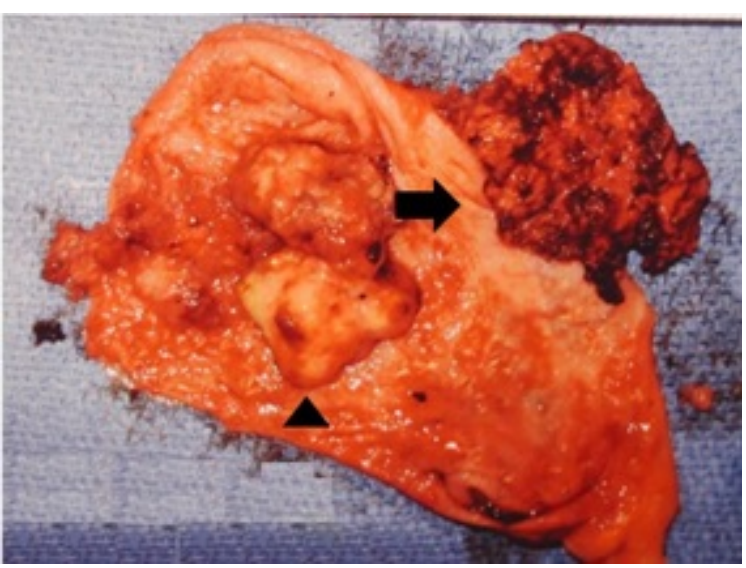

Figure 1: Gallbladder with polypoid mass (black arrowhead) and irregularly attached hepatic parenchyma (black arrow) associated with a significantly thickened gallbladder wall.

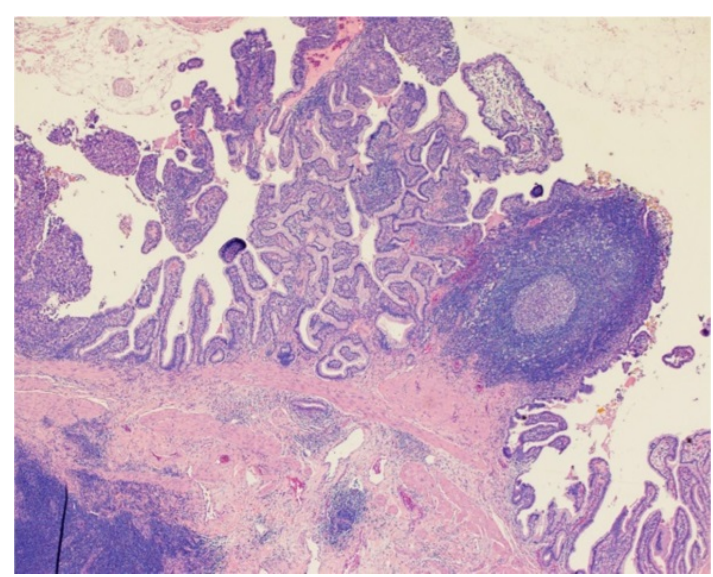

Figure 2: Histologic sections of the mass consisted of a papillary neoplasm with a reactive mucosal lymphoid follicle.

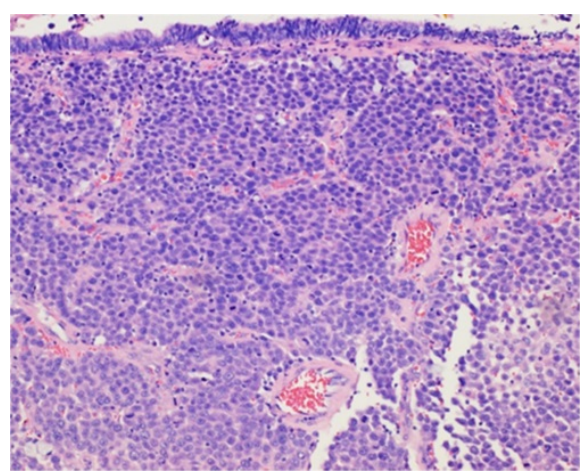

Figure 3: Sections of the thickened gallbladder wall show sheets of invasive poorly differentiated malignant neoplastic cells. The gallbladder wall is covered by dysplastic columnar epithelium.
Histologic sections of the mass revealed a papillary neoplasm covered by variably dysplastic columnar epithelial cells associated with occasional prominent reactive mucosal lymphoid follicles (Figure 2). The underlying gallbladder wall was infiltrated by poorly differentiated malignant neoplastic cells, extending virtually through the full thickness of the gallbladder wall (Figure 3). The neoplastic cells were moderately pleomorphic with vesicular nuclei, prominent nucleoli, and occasional atypical mitoses (Figure 4). Some neoplastic glands were surrounded by the poorly differentiated neoplastic cells (Figure 5). The attached resected portions of the liver parenchyma revealed moderate hepatic steatosis with no evidence of malignancy.

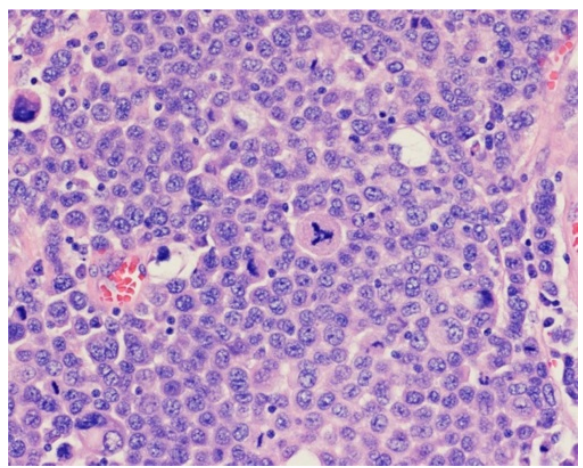

Figure 4: The poorly differentiated malignant neoplastic cells showed moderately pleomorphic vesicular nuclei with prominent nucleoli and atypical mitoses.

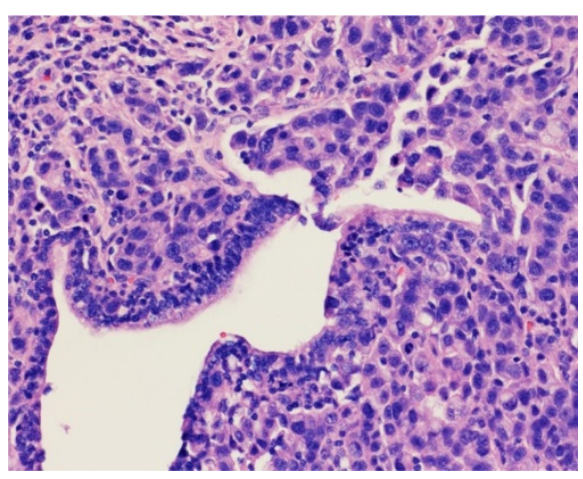

Figure 5: In some areas, there is a mixture of well-differentiated mucinous glandular epithelial neoplasm surrounded by sheets of poorly differentiated malignant neoplastic cells.

Immunohistochemical studies showed strongly positive staining of both the papillary and undifferentiated invasive malignant cells for pan-CK, CK20, and S100. The invasive malignant cells were, however, also positive for neuron-specific enolase and chromogranin (Figure 6), but negative for both synaptophysin and CD56. All neoplastic cells, papillary and invasive undifferentiated components, stained strongly positive for $\mathrm{p} 53$. 
Citation: Parsa C, Orlando R, Narayanan K, Duarte D, Webb S, (2018) Primary Mixed Adenoneuroendocrine Carcinoma (MANEC) of Gallbladder, Report of Two Cases with Different Histologic and Immunohistochemical Features. J Tumor Res \& Reports 3: 120.

Page 3 of 5

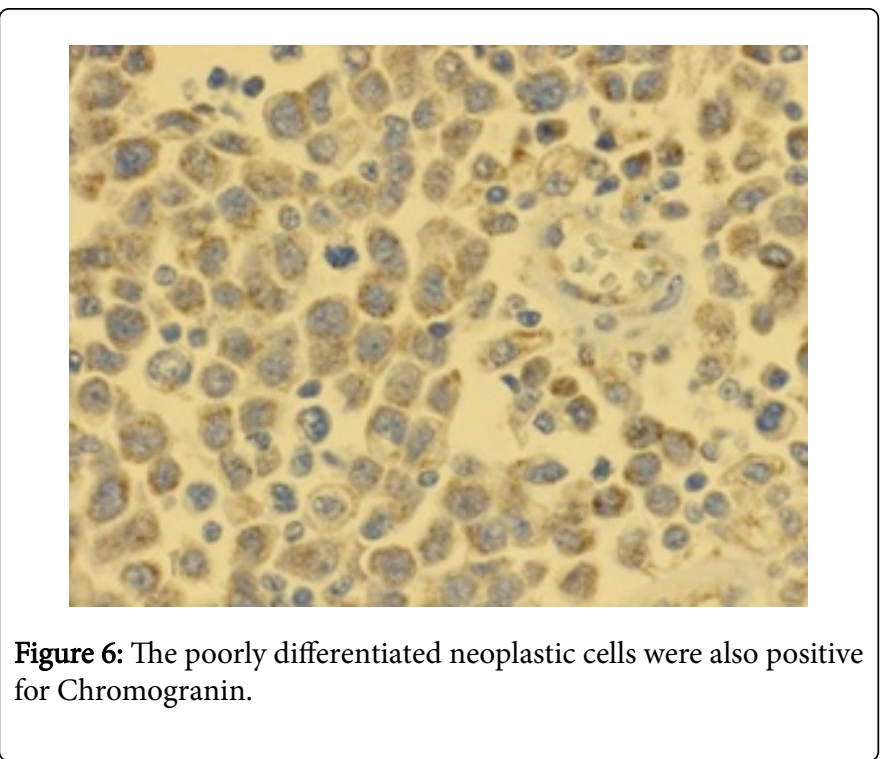

\section{Case 2}

A 76-year-old male with past medical history of cholelithiasis and diabetes presented to the emergency department after several days of worsening abdominal pain, fever, chills, and diaphoresis. Over the previous 3-4 days, the patient experienced worsening right-sided abdominal pain and generalized weakness with associated "yellowing" of his skin. The patient was told he had gallstones in the past, which had intermittently caused him pain. For relief, the patient had tried herbal supplements and other home remedies. He admitted to having had thyroid biopsies for possible thyroid disease. His past medical history was significant for a known systolic heart murmur, gallstones, hypertension, open angle glaucoma, hemoptysis, and bronchiectasis.

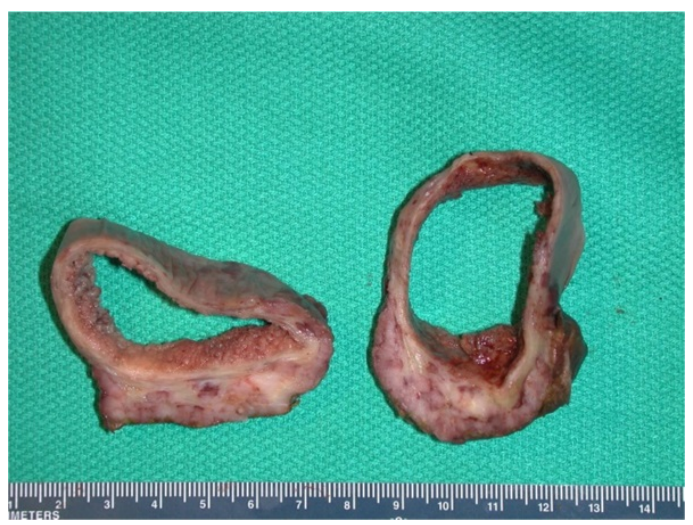

Figure 7: Cross-section of gallbladder in the second case shows a significantly and asymmetrically thickened gallbladder wall with a markedly roughened red-brown mucosal surface.

On physical examination, scleral icterus and frank jaundice of the skin was apparent. The abdomen, apart from obesity, was slightly distended, and tender to palpation in the right upper and lower quadrants, without rebound tenderness. The patient's clinical signs and symptoms were consistent with acute cholecystitis with possible septic shock. He was subsequently started on antibiotics.

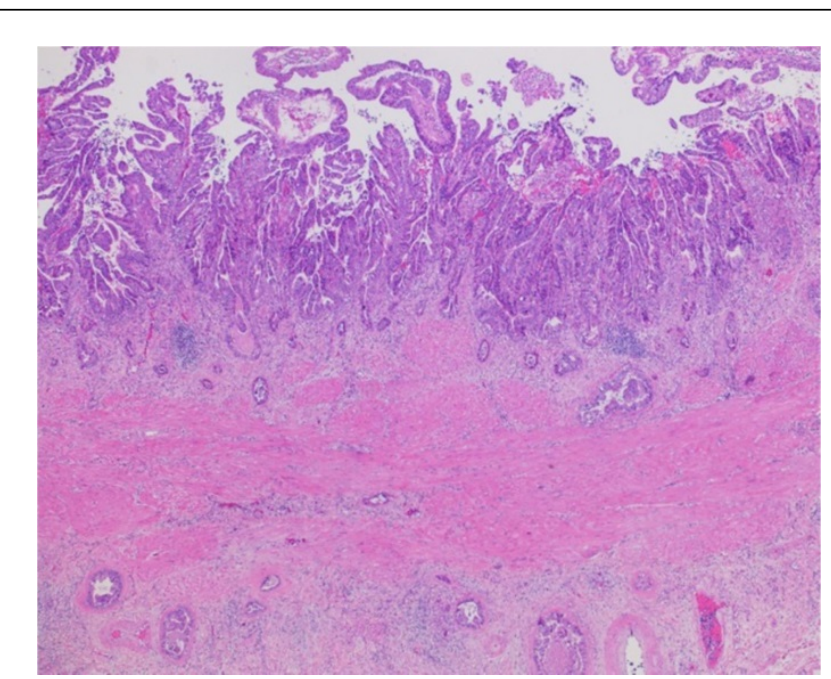

Figure 8: In this area, the gallbladder mucosa is replaced by dysplastic epithelial cells with few malignant neoplastic glands extending through the full thickness of the gallbladder wall.

Computed tomography (CT) scan of abdomen revealed a distended gallbladder with wall thickening. The common bile duct was not distended, but there was suspected intrahepatic biliary dilatation. The CT findings were consistent with cholecystitis. Abdominal ultrasound revealed a $7 \mathrm{~mm}$ thickened gallbladder wall with pericholecystic edema. The spleen, liver, pancreas, and adrenal glands appeared normal. The prostate, however, appeared markedly enlarged. There were no enlarged lymph nodes within the abdomen or pelvis. A chest CT revealed areas of air and soft tissue attenuation in the region of the lingula compatible with the pulmonary fibrosis with honeycombing. An open cholecystectomy was performed the following day with intraoperative concerns for possible gangrenous cholecystitis.

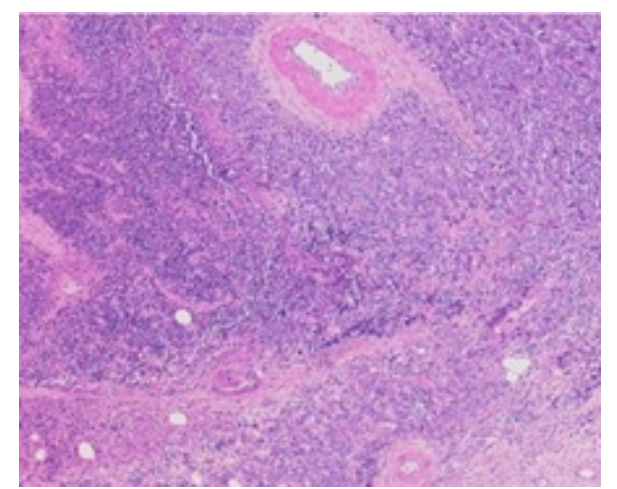

Figure 9: H\&E section of gallbladder wall infiltrated by closely packed poorly differentiated malignant neoplastic cells with few neoplastic glands.

The removed gallbladder was $12.0 \mathrm{~cm}$ long and $5.0 \mathrm{~cm}$ in diameter. The serosal surface was covered partially by roughened pink-brown serosa. On section, the lumen of the gallbladder was filled with 
Citation: Parsa C, Orlando R, Narayanan K, Duarte D, Webb S, (2018) Primary Mixed Adenoneuroendocrine Carcinoma (MANEC) of Gallbladder, Report of Two Cases with Different Histologic and Immunohistochemical Features. J Tumor Res \& Reports 3: 120.

Page 4 of 5

gelatinous dark-red hemorrhagic material and multiple smooth redbrown calculi of variable sizes, measuring up to $3.0 \mathrm{~cm}$ in diameter. The wall of the gallbladder varied from $0.3 \mathrm{~cm}$ to $2.0 \mathrm{~cm}$ in thickness (Figure 7). The thickened gallbladder wall, resembling a mass, measured $7.0 \times 4.0 \mathrm{~cm}$ in greatest dimensions.

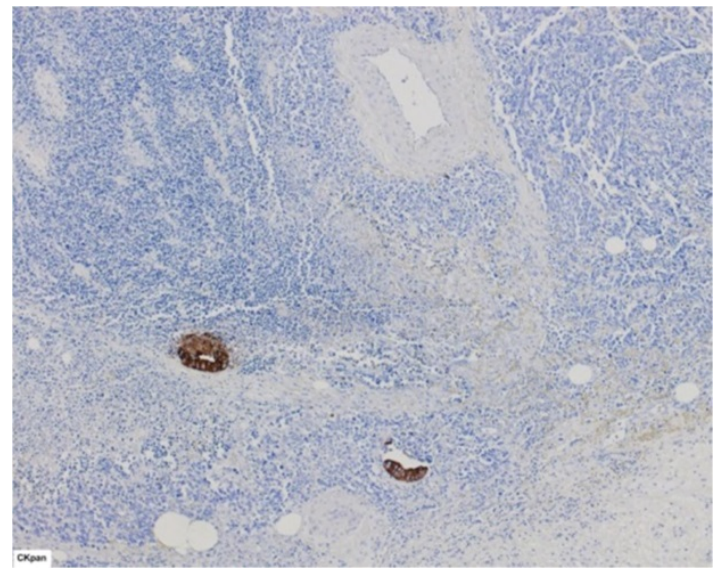

Figure 10: Pan-CK of the same area as figure 9 highlighting only the neoplastic glands. In some areas, the poorly differentiated neoplastic cells virtually merged with the neoplastic glandular epithelial cells.

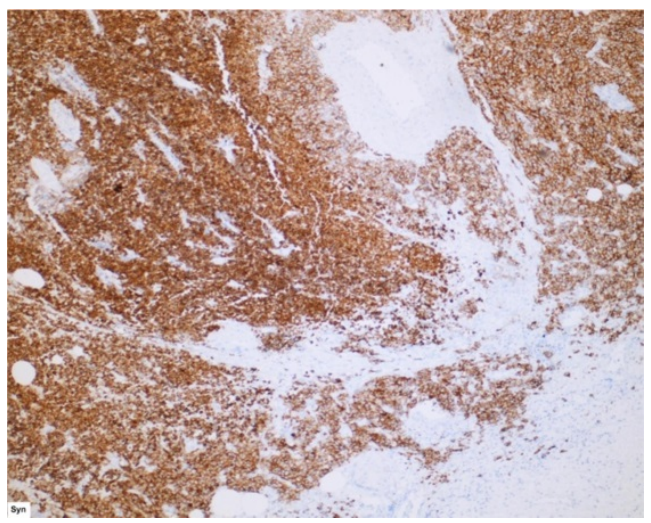

Figure 11: Synaptophysin immunostain of same area as figure 10 highlights only the poorly differentiated malignant neuroendocrine cells.

Histologic sections of the thickened gallbladder wall showed papillary mucosal surface covered by dysplastic epithelium with scattered malignant neoplastic cells extending deep into the gallbladder wall (Figure 8). Poorly differentiated malignant neoplastic cells were also discernible in portions of the gallbladder, infiltrating deep into the wall, occasionally surrounding few of the neoplastic glands (Figure 9). The margins, on the hepatic side of the gallbladder and the cystic duct, were also found to be involved by the invasive carcinoma. Additional pathologic findings included severe chronic cholecystitis and multiple mixed dark-brown calculi with intraluminal haemorrhage.
On Immunohistochemical studies, the neoplastic glands were negative for neuroendocrine markers, but positive for pan-CK; the poorly differentiated neoplastic cells were positive for neuroendocrine markers, and negative for pan-CK (Figure 10). The poorly differentiated neoplastic cells were focally positive for chromogranin and diffusely positive for synaptophysin (Figure 11). In some areas, the poorly differentiated neoplastic cells virtually emerged with the neoplastic glandular epithelial cells (Figure 12). A separately submitted specimen, labelled from the "diaphragm" consisted exclusively of the poorly differentiated neuroendocrine component.

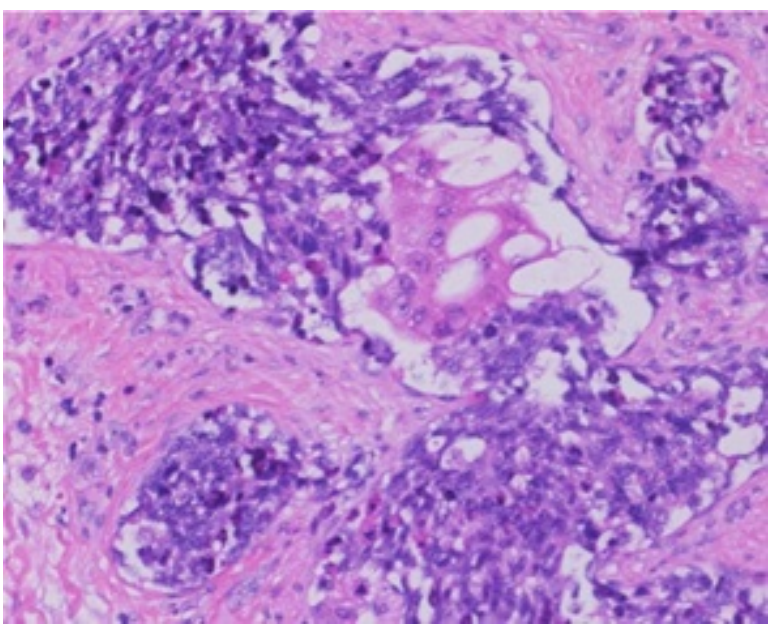

Figure 12: High power section of an area showing neoplastic glands virtually merging with the neoplastic neuroendocrine cellular component.

\section{Discussion}

Mixed adenoneuroendocrine carcinoma (MANEC), has been described by WHO as a rare gastrointestinal neoplasm [1], histologically characterized by the presence of at least $30 \%$ each of epithelial and neuroendocrine components [2]. The tumor may appear in various levels of the digestive tract including the esophagus, stomach, colon and appendix [3-7], as well as other sites such as the pancreas [8], and urinary bladder [9-12]. Presence of MANEC in the gallbladder is exceedingly rare [2,13-15]. Biological behaviour of MANEC seems to be quite unpredictable and the prognosis uncertain. Paucity of neuroendocrine cells in normal gallbladder has led to speculations regarding possible origins regarding MANEC [2]. The pathogenesis of this neoplasm may involve intestinal metaplasiadysplasia-carcinoma with synchronous or asynchronous progression to an epithelial and a neuroendocrine malignancy in the gallbladder. The latter is based on cases of MANEC in gallbladders with extensive intestinal metaplasia. Another pathway in the development of MANEC may involve a neoplastic stem cell with potential to differentiate along multiple cell lines with epithelial and neuroendocrine features. A chronically inflamed microenvironment, present in all the reported cases, is the most likely initial predisposing event in this mutational, metaplastic or stem cell, transformation process.

The two cases presented in our article showed interesting differences in their histologic and immunohistochemical findings. Both cases demonstrated significant neoplastic epithelial and neuroendocrine 
Citation: Parsa C, Orlando R, Narayanan K, Duarte D, Webb S, (2018) Primary Mixed Adenoneuroendocrine Carcinoma (MANEC) of Gallbladder, Report of Two Cases with Different Histologic and Immunohistochemical Features. J Tumor Res \& Reports 3: 120.

Page 5 of 5

components to warrant a diagnosis of MANEC. First case with a welldifferentiated papillary epithelial neoplasm with a poorly differentiated neuroendocrine component, while in the second case both neoplasms were poorly differentiated with characteristic epithelial and neuroendocrine immunohistochemical features. In our first case, the poorly differentiated neoplastic cells were positive for pancytokeratin, chromogranin, and neuron-specific enulase (NSE), but were negative for synaptophysin. In our second case, the poorly differentiated neoplastic cells were negative for pancytokeratin, but strongly positive for synaptophysin. In the latter case, the neoplastic epithelial and neuroendocrine cells seemed to focally merge with one another, suggestive of same stem cell of origin differentiating or dedifferentiating into different phenotypes.

These tumors may be stratified into different prognostic categories based on their clinical presentation, findings at imaging studies, histologic grade, and TNM cancer staging applications. Recent studies suggest that treatment should be guided by the most aggressive histologic component. Although treatment options have not been standardized, cholecystectomy, possibly followed by multimodal anticancer therapies, may be beneficial.

\section{References}

1. Bosman FT, Carneiro F, Theise ND (2010) Nomenclature and classification of neuroendocrine neoplasms of digestive system. WHO Classification of Tumors of the Digestive System. (4thedn) IARC, Lyon $13-24$.

2. Acosta AM, Wiley EL (2016) Primary Biliary Mixed Adenoneuroendocrine Carcinoma (MANEC) A Short Review. Arch Pathol Lab Med 140: 1157-1162.

3. Vanacker L, Smeets D, Hoorens A, Teugels E, Algaba R, et al. (2014) Mixed Adenoneuroendocrine carcinoma of the colon: molecular pathogenesis and treatment. Anticancer Res 34: 5517-5522.

4. Liu XJ, Feng JS, Xiang WY, Kong B, Wang LM, et al. (2014) Clinicopathological features of an ascending colon mixed adenoneuroendocrine carcinoma with clinical serosal invasion. Int J Clin Exp Pathol 7: 6395-6398.

5. Gurzu S, Kadar Z, Bara T, Bara TJ, Tamasi A, et al. (2015) Mixed adenoneuroendocrine carcinoma of gastrointestinal tract: report of two cases. World J Gastroenterol 21: 1329-1333.

6. Zhang W, Xiao W, Ma H, Sun M, Chen H, et al. (2014) Neuroendocrine liver metastasis in gastric mixed adenoneuroendocrine carcinoma with trilineage cell differentiation: a case report. Int J Clin Exp Pathol 7: 6333-6338.

7. Šefr R, Němec L, Fabian P, Fiala L (2017) Mixed adenoneuroendocrine carcinoma (MANEC) of the gastrointestinal tract. Rozhl Chir Winter 96: 41-44.

8. La Rosa S, Marando A, Sessa F, Capella C (2012) Mixed adenoneuroendocrine carcinomas (MANECs) of the gastrointestinal tract: an update. Cancers (Basel) 4: 11-30.

9. Gurzu S, Kadar Z, Bara T, Bara TJ, Tamasi A, et al. (2015) Mixed adenoneuroendocrine carcinoma of gastrointestinal tract: Report of two cases. WJG 21: 1329-1333.

10. Murata M, Takahashi H, Yamada M, Song M, Hiratsuka M (2017) A case of mixed adenoneuroendocrine carcinoma of the pancreas. Medicine 96: 9.

11. Pham QD, Mori I, Osamura RY (2017) A Case Report: Gastric Mixed Neuroendocrine-Nonneuroendocrine Neoplasm with Aggressive Neuroendocrine Component. Hindawi Case Reports in Pathology 2017: $1-6$.

12. Eckstein M, Rasper B, Busche J, Stachetzki U, Wistuba DE, et al. (2017) Primary mixed adeno-neuroendocrine carcinoma (MANEC) of the urinary bladder a rare entity. Aktuelle rol 48: 350-355.

13. Mahansaria SS, Agrawal N, Arora A (2017) Ampullary Mixed Adenoneuroendocrine Carcinoma: Surprise Histology, Familiar Management. International Journal of Surgical Pathology 25: 585-591.

14. Chen H, Shen YY, Ni XZ (2014) Two cases of neuroendocrine carcinoma of the gallbladder. WJG 20: 11916-11920.

15. Huang Z, Xiao WD, Li Y, Huang S, Cai J, et al. (2015) Mixed adenoneuroendocrine carcinoma of the ampulla: two case reports. WJG 21: 2254-2259. 